\title{
La question basque au miroir de la violence
}

\section{Daniel Hermant}

\section{(2) OpenEdition}

1 Journals

\section{Édition électronique}

URL : http://journals.openedition.org/conflits/660

DOI : $10.4000 /$ conflits.660

ISSN : $1777-5345$

Éditeur :

CCLS - Centre d'études sur les conflits lilberté et sécurité, L'Harmattan

\section{Édition imprimée}

Date de publication : 22 octobre 1992

ISSN : 1157-996X

\section{Référence électronique}

Daniel Hermant, «La question basque au miroir de la violence », Cultures \& Conflits [En ligne], 07 | automne 1992, mis en ligne le 06 janvier 2003, consulté le 30 mars 2021. URL : http:// journals.openedition.org/conflits/660; DOI : https://doi.org/10.4000/conflits.660

Ce document a été généré automatiquement le 30 mars 2021.

Creative Commons License 


\title{
La question basque au miroir de la violence
}

\author{
Daniel Hermant
}

La série d'attentats sanglants d'ETA du début de l'année ${ }^{1}$, la capture le 29 mars 1992, de son chef, Artapalo, et a contrario la proposition de trêve de deux mois des attentats faite le 10 juillet par cette organisation, puis leur reprise le 17 août, amènent une fois de plus l'observateur à s'interroger sur la violence en Espagne et sur l'évolution du problème basque et ce, d'autant que pour beaucoup de spécialistes, l'année 1992 est à bien des égards une année décisive pour ETA.

Si effectivement personne ne peut deviner les choix que fera ETA une fois sa direction reconstituée -c'est quand même eux qui conditionneront l'arrêt ou la poursuite de la violence - il est par contre possible d'examiner le cadre dans lequel, en tout état de cause, la stratégie d'ETA se déploiera, et il est utile de se demander si ce cadre qui reste structurant si l'on réfléchit en terme d'efficacité politique, a conservé une certaine stabilité depuis la reprise de l'activité d'ETA, c'est-à-dire depuis une vingtaine d'années environ, ou si un glissement continu n'aurait pas modifié, sans qu'on y prenne garde, la position du mouvement national basque en Espagne et sapé les bases de l'organisation clandestine.

Perspectives policières

Un premier critère d'appréciation repose sur l'analyse des résultats de la police. Ceuxci sont jugés très encourageants par beaucoup d'observateurs. 1991 a vu le démantèlement d'une dizaine de commandos et une pression policière accrue en France pour isoler les refugiados du reste du mouvement, l'année 1992 a commencé également par des succès policiers considérables. Sur un plan organisationnel, beaucoup pensent que le mouvement se trouve confronté à des difficultés de recrutement qui se traduisent par une baisse du niveau d'efficacité ainsi qu'à des problèmes de nature logistique et financière comme l'indiquent les réactions très vives d'Herri Batasuna lors du démantèlement en janvier 1992 d'un réseau de racket d'industriels. Dans ce contexte, ETA - dit-on - doit restreindre ses aides aux familles des prisonniers, et aurait même conseillé à ses membres de diminuer leurs déplacements 
pour des raisons financières! Ces éléments réconfortants pour les autorités s'expliquent par une plus grande efficacité de la police et de la garde civile. Il faut y ajouter la montée en puissance de l'Ertzaintza, la police autonome basque, qui a joué un rôle direct dans le démantèlement de commandos en août de l'année dernière ou plus récemment en janvier 1992, dans la mise en évidence de la participation de membres d'Herri Batasuna au racket financier pro-ETA ${ }^{2}$. Enfin la collaboration avec la police française s'améliore comme le montre l'arrestation d'Artapalo en France.

Ce discours n'est pas totalement neuf, les autorités policières le tiennent périodiquement, il est balancé par une autre analyse plus pessimiste disant que quels que soient les succès de la police - et ils furent grands pendant les six premiers mois de 1992- mesurer leurs effets sur les capacités d'action du mouvement est aléatoire puisque faire des attentats ne demande en définitive que très peu de moyens et que de toute manière, il n'y a pas de solution uniquement policière un problème de cette nature.

A ces hypothèses sur l'évolution du combat policier, il faudrait ajouter une remarque plus générale. L'entrée en lice de l'Ertzaintza modifie les conditions générales de la lutte policière. Basque, ce corps de police autonome, après avoir été regardé avec méfiance par l'opinion espagnole, est entré franchement dans la lutte contre ETA. Certes, on peut toujours s'interroger sur l'autonomie d'un corps de police dont les chefs sont encore espagnols, certes la politique de retrait de la police nationale du Pays basque n'est pas encore totale bien en qu'elle ait commencé, et il n'est pas question que la garde civile lâche le contrôle des frontières. Force est cependant de noter que l'appréciation portée sur l'Ertzaintza a radicalement changé et que la question d'actualité n'est plus celle de son noyautage par ETA comme c'était le cas lors de sa fondation, mais celle de sa confrontation avec ETA ${ }^{3}$.

Une confrontation avec la police autonome mettrait en porte-à-faux le mouvement séparatiste et montrerait avec évidence la rupture représentativité de l'organisation en faisant s'effondrer d'un bloc la rhétorique patiemment construite sur la segmentation de la lutte entre peuple basque et État central espagnol: comment dénoncer la répression policière ou militaire du pouvoir central si ETA s'en prenait à des policiers basques? Certes, la vision d'un Pays basque divisé entre deux camps, sur un modèle quasi-irlandais, est une hypothèse extrême, à notre avis peu probable, mais il faut l'avoir présente à l'esprit pour envisager l'avenir de la violence ${ }^{4}$. S'il convient de rester très prudent dans ces affirmations, on peut néanmoins dire que la situation connaît une évolution structurelle avec l'arrivée et la montée en puissance d'un nouveau partenaire l'Ertzaintza. Cette évolution a rendu possible une série de coups très durs contre ETA et son infrastructure. Si les succès de la police n'ont pas eu jusqu'à présent comme conséquence une fuite en avant de l'organisation dans la violence, il ne faut pas pour autant exclure cette possibilité, car l'expérience montre que l'évolution à moyen terme vers la criminalité des membres d'une organisation clandestine provoque souvent à court terme un pic de violence politique.

Perspectives stratégiques

Sur le plan stratégique, d'autres calculs peuvent être faits et ce quelle que soit la situation "policière" d'ETA. Le duel des volontés le désir de faire enfin plier l'ennemi focalise une présentation stratégique du problème selon laquelle la politique étant le débouché naturel du militaire, la pression violente peut tout faire basculer. Si la logique déployée est celle d'une violence de type militaire qui fonctionne sur la rupture, le 
retournement, l'effondrementS, et dont l'objectif défini par Clausewitz est "l'anéantissement de la volonté de l'ennemi", alors l'année 1992 présentait une configuration particulièrement intéressante. Les jeux Olympiques, l'Exposition universelle de Séville et l'anniversaire de la découverte de l'Amérique - toutes manifestations de masse largement médiatisées - pouvaient offrir des moyens de pression particulièrement forts sur le gouvernement espagnol, car une série d'attentats menée contre ces manifestations aurait eu un effet dévastateur dans l'opinion mondiale pour l'image de marque de l'État espagnol. Une hypothèse fréquemment avancée consistait à dire qu'ETA allait utiliser cette conjoncture exceptionnelle pour forcer le gouvernement espagnol à s'asseoir à la table des négociations et obtenir enfin les concessions qui lui ont été refusées à Alger. On comprend que cette perspective abondamment commentée dans le pays pendant toute l'année ait constitué pour les autorités de Madrid une source de graves préoccupations.

Cette présentation en terme stratégique reposait sur le postulat d'un usage rationnel de la violence optimisée par une organisation qui profite d'une conjoncture particulièrement favorable. Elle supposait un planning d'exécution: la manoeuvre (pour reprendre un terme "militaire") devant être exécutée avant le 13 octobre, puisque après cette date, il n'existera plus d'échéance spectaculaire. Elle focalisait sur l'idée d'un marchandage violent où la pression, la menace d'attentats, mèneraient le gouvernement espagnol à des concessions décisives pour éviter un camouflet médiatique. Le résultat de la confrontation actuelle dépendant du rapport entre les capacités de "frappe" d'ETA et celles d'encaissement" du gouvernement espagnol.

On peut spéculer à l'infini sur les raisons stratégiques qui ont amené ETA à renoncer à ces calculs, mais il vaut peut-être mieux élargir le cadre d'analyse et constater qu'il n'existe pas un rapport simple entre le niveau de violence d'une "pression" et la volonté "négociatrice" de celui à l'égard duquel elle s'exerce : un accroissement de la violence n'offre pas nécessairement une chance de plus de faire céder l'adversaire, au contraire un excès peut provoquer un retournement de position. Il y a une illusion sur la dimension linéaire de la violence, sur le parallélisme entre la montée des enjeux et l'ampleur des conséquences. Aussi, s'il est possible que ces calculs liés à des anticipations des acteurs soient réels, ils ne rendent pourtant que très imparfaitement compte de la manière dont est produite la violence en Espagne et les effets qu'elle engendre. Faire ces calculs revient à raisonner comme si la violence occupait et structurait toute la scène politique en Espagne, ce qui suppose que celle-ci soit dominée par les rapports de répulsion/fascination/mobilisations que génère la violence. Or il n'en est rien, la violence est, fort heureusement, marginale en Espagne et n'a pas le même impact au Pays basque et dans le reste de la nation. Fonder une politique sur les effets de la violence est extrêmement aléatoire ; la scène stratégique est loin d'être une arène vide : à côté d'ETA et du gouvernement espagnol, il faut au moins tenir compte des réactions de l'opinion (basque ou espagnole) et de celles de la classe politique.

L'analyste doit donc resituer ces discussions dans le jeu politique et non les en extraire selon une logique de "guerre", d'"urgence" qui n'est que la reprise du discours idéologique d'ETA ou des organisations révolutionnaires sur l'effet "transformateur, mobilisateur, fusionnel" de la violence.

Scène politique espagnole

Les effets du jeu politique sur la violence peuvent parfaitement être saisis en suivant les réactions provoquées par les attentats. Chaque attentat suscite des déclarations et une 
inflation verbale qui occupent l'ensemble des acteurs politiques avec des jeux d'escalade très significatifs. Après les critiques de la presse, l'opposition renchérit sur l'inefficacité policière du gouvernement dans le cadre d'une description laxisme/ fermeté, mais le gouvernement, s'il est responsable, ne doit pas se laisser aller à des déclarations maximalistes qui favoriseraient la politique du pire : provocations d'ETA répression gouvernementale, cycle de la violence. Le mimétisme des déclarations est facilité par l'existence d Herri Batasuna qui sert de "porte-parole" à ETA, comme on a $\mathrm{pu}$ le constater une fois encore lors de la querelle à propos de l'implication des dirigeants d'Herri Batasuna dans la levée de l'impôt révolutionnaire d'ETA 5 .

La tentation de satisfaire une partie de l'opinion par des déclarations faciles, mais ne faisant pas avancer la question d'un pouce, nous place au centre de la scène politique espagnole, où le sort du Pays basque n'occupe qu'un espace limité. Sur ce plan, la querelle se développe sur les moyens "politiques" d'éradiquer ETA. Comme il n'est pas possible de jouer à court terme sur l'appui populaire dont dispose ce mouvement au Pays basque, la polémique porte essentiellement sur la répression contre Herri Batasuna, et s'alimente dans des reconstitutions de l'organigramme secret d'ETA - Qui appartient à la "cúpula" ? - dont l'objet est de prouver la politique de double jeu du personnel politique d'Herri Batasuna ${ }^{6}$. Cette attitude conforte l'attitude abertzale à qui elle fournit par le biais de la stigmatisation une confortable identité, doublée d'une position de martyre.

Le dernier volet de la querelle porte sur la situation des rapports policiers avec la France. Cette question est un moyen commode de réconcilier les acteurs espagnols en faisant porter la responsabilité des échecs sur l'extérieur: c'est l'inefficacité de la France qui est à l'origine, dit-on, de la vitalité d'ETA. Cela permet également des effets d'annonce, comme au début février, avec la parution télécommandée dans la presse espagnole, après l'attentat de Madrid, d'une information selon laquelle une brigade mixte franco-espagnole allait être créée - information démentie quinze jours après par la France ${ }^{7}$. Ce mode de gestion du terrorisme, qui n'est pas sans rappeler nos propres pratiques, - en France - correspond à des mécanismes de la vie politique, somme toute assez ordinaires, mais suffisamment contraignants pour rendre impossible les retournements spectaculaires.

S'il existe un effet de résonance de la violence sur l'action des acteurs politiques, cet effet n'est pas direct, mécanique, il ne dépend pas des objectifs des acteurs violents, mais des calculs ou des interprétations dans lesquels la lutte contre l'organisation n'est pas primordiale: la violence politique est instrumentalisée par les organisations politiques pour prendre avantage sur les concurrents politiques.

Scène politique basque

Crise du discours radical basque

Le mouvement national basque s'appuie sur un discours intégrant la dimension nationale et la dimension sociale ou économique qui légitime une stratégie de lutte vers l'indépendance. "Nos idées ont-elles vieilli ?" s'interroge Herri Batasuna en constatant "l'offensive de l'idéologie dominante". Ce constat pudique de la perte de crédibilité du discours abertzale de plus en plus décalé, dans ses concepts et son vocabulaire, d'un monde décidément bien changeant, a entraîné un effort de "rénovation théorique". Il a été décidé en 1988 par Herri Batasuna de réévaluer les perspectives du mouvement tous les trois ans et d'en adapter si nécessaire les structures organisationnelles. En 1991, sous le titre "Urrats Berri", une série de quatre documents a été rédigée par Herri 
Batasuna pour mettre au goût du jour son discours et définir le cadre de son action ${ }^{8}$. Mais en analysant ces documents, on ne peut qu'en voir le style marxiste désuet et le caractère sommaire : aucune analyse sérieuse n'est faite, ni sur le plan culturel, ni sur le plan économique et social.

Sur le plan culturel, si le discours national est, comme toutes les réflexions en bonne d'approche culturaliste, immuable dans son affirmation identitaire, la dénonciation de l'oppression imposée par le gouvernement espagnol qui en constitue la dimension combative apparait à l'heure actuelle comme bien rituelle dans une région régie depuis dix ans par un statut d'autonomie. Il existe sur le point crucial de la politique linguistique, un décalage entre le libéralisme qui entoure les pratiques quotidiennes de la population basque et le discours de protestation et revendication systématiques de la gauche abertzale. En la matière, la querelle linguistique poste moins sur la possibilité d'utiliser le basque pour ceux qui le désirent que sur le moyen de l'imposer9.

Les analyses sur la stratégie industrielle de l'État central épaulé par les multinationales qui constituent le deuxième thème général du mouvement, paraissent également bien anachroniques dans leur dénonciation de la nature coloniale et anti-ouvrière de la politique industrielle que mènerait l'État espagnol au Pays basque. On peut en conclure que non seulement le chantier, pour définir un nouveau modèle économique et social, n'est pas ouvert dans ces textes, mais que la maitrise intellectuelle dans ce domaine a reculé, car aux querelles qui ont toujours opposé ceux qui pensaient d'abord en termes de classe (qualifiés d'espagnolistes) et ceux pour qui la structuration nationale restait même sur ce plan primordiale, s'est ajouté l'effondrement du communisme à l'Est. Aussi la gauche abertzale en panne de modèle de société se contente-t-elle depuis plusieurs années d'une dénonciation plus ou moins aigre de la gestion madrilène, ou même de celle du PNV au sein du gouvernement autonome, à laquelle elle attribue (directement ou indirectement) la responsabilité de l'effondrement des industries traditionnelles au Pays basque et la crise sociale.

Le volet de l'action proprement politique est plus complexe car il ne se réduit pas simplement à un discours, il est éclaté entre la gestion des affaires au sein des institutions autonomes basques auxquelles participe la coalition d'Herri Batasuna à travers ses élus, et la pratique de la pression violente par ETA. Herri Batasuna est partagée entre les contacts permanents qu'elle est obligée de maintenir comme organisation politique, et le soutien à ETA qu'elle formule à travers un discours "rupturiste". Le refus de l'appellation de "parti politique" au profit de celle "d'alliance tactico-stratégique" n'a fait que souligner par le vocabulaire cette contradiction interne. Cet écartèlement entre le pôle réformiste et le pôle révolutionnaire, pour reprendre le vocabulaire du mouvement abertzale, rend incommode la vie des membres d'Herri Batasuna et leur impose une casuistique du sens très complexe. Il leur faut distinguer soigneusement les niveaux institutionnels (local, régional, national, européen), la nature de leur participation (permanente, non sur la permanente, occasionnelle, exceptionnelle) et justifier à chaque fois cette attitude par un objectif politique. L'image simplificatrice de la courroie de transmission que serait Herri Batasuna pour ETA, rend très imparfaitement compte de ces tensions entre deux logiques d'action et des flottements ou les hésitations du mouvement nationaliste que cette tension entraîne au sein de son aile radicale ${ }^{10}$.

Du côté d'ETA les choses ne sont pas simples également. Le choix de la violence comme mode d'action principal de l'organisation a entraîné au fil des années une succession de 
scissions entre "polimili"11 et "milis" ${ }^{12}$ qui sont toujours traduites par la disparition des modérés, sans pour autant que les bénéfices politiques tirés de cette stratégie soient évidents. Or, les interférences entre ces deux lignes sont plus que jamais d'actualité comme le montre les tous récents mouvements de prisonniers, complaisamment mis en avant par le gouvernement espagnol. Fait sans précédent pour des militants qui se considéraient comme de "vrais soldats", plus de $40 \%$ d entre eux se désolidariseraient de la ligne dure d'ETA et voudraient une négociation avec le gouvernement espagnol qui améliorerait leur sort. Les voies pour atteindre l'indépendance, longtemps réduites au modèle proposé par ETA, ne sont-elles pas en train de se diviser?

Le désir d'indépendance : $\mathrm{d}$ 'un modèle militaire à un modèle gestionnaire

Depuis la reprise de ses activités, il y a une vingtaine d'années ETA fonctionne selon un modèle de gestion militaire de l'indépendance. Ce modèle trouve son origine dans les conditions historiques de l'Espagne et de l'époque. La lutte contre le franquisme est à la fois la dernière lutte contre le fascisme historique et une lutte de libération nationale qui se moule sur les guerres de décolonisation de la période. En 1974, l'attentat contre l'amiral Carrero Blanco ressenti comme fortement légitime bien au-delà du Pays basque a probablement marqué le sommet de ce type de conjoncture historique. Même si les temps ont changé, l'indépendance est restée pour les Basques un objectif hautement symbolique et mobilisateur. Les manifestations autour du drapeau, des morts, font renaître immédiatement un sentiment de fraternité. Bien sûr l'Espagne n'est plus fasciste et la passion nationaliste s'est érodée comme l'avait fait avant elle la passion religieuse, mais une sorte de nationalisme de crise perdure. C'est lui qui explique les grandes manifestations de soutien à ETA dès que cette organisation paraît menacée par la répression du pouvoir. Bien sûr la garde civile de l'ère Felipe Gonzalez n'a plus grand chose à voir avec celle du franquisme, mais au Pays basque elle cristallise encore assez facilement la haine et permet des comportements fusionnels dans la grande unité mythique de la nation.

Sur un plan plus politique, cette légitimité du modèle militaire s'est maintenue à la fois parce que le mouvement nationaliste vivait sur un stock d'analyses issu d'une situation internationale qui n'existe plus guère, mais également parce que le mode de fonctionnement organisationnel a toujours sélectionné en priorité des dirigeants adoptant un répertoire d'action violente ${ }^{13}$.

En face de ce modèle militaire, un second modèle existe - appelons-le gestionnaire. Pour ses promoteurs, réduire l'indépendance à un choix politique accessible d'un bloc par une décision du gouvernement espagnol et par dessus la tête des partis politiques, relève de l'inconséquence car c'est faire de la constitution espagnole et du bon sens politique, et ignorer la complexité des liens (industriels, infrastructures, administratifs ou politique de délimitation de compétence) qui amarrent le Pays basque à l'ensemble national ${ }^{14}$. Aussi, dans cette hypothèse, situer le Pays basque dans l'ensemble espagnol ne relève pas du jeu de bascule mais exige une évolution statutaire complexe entre le gouvernement autonome basque et le gouvernement espagnol, car l'indépendance devient moins, dans cette optique, un objet à conquérir qu'un objet à construire. Elle ne renvoie pas à une démarche "militaire", mais à une démarche "politique" où l'objectif à atteindre prend forme à travers des compromis entre moyens et fins. Arriver à l'indépendance ne peut se faire que par une exploitation continue et patiente du statut d'autonomie au Pays basque, ou en utilisant les moyens juridiques politiques existant dans le cadre européen. Seul un parti modéré, bien implanté, comme le PNV et de surcroît parti nationaliste historique, qui a été capable de gérer avec le gouvernement 
espagnol la mise en place et l'évolution du statut d'autonomie de Guernica, est capable de faire bouger les choses. Mais cette dimension procédurale et dynamique de l'action du PNV laisse assez flou le contenu de l'indépendance si cet objectif est maintenu ${ }^{15}$.

Évolutions possibles

Une marge de manoeuvre étroite

L'étroitesse de la marge de manoeuvre peut d'abord être résumée à travers l'examen des déchirements d'Herri Batasuna: organisation politique faisant campagne électorale, participant à la gestion des affaires basques, proclamant que le dialogue et la négociation sont seuls capables de dépasser la violence actuelle, mais en même temps, mouvement soutenant inconditionnellement ETA, refusant de condamner l'usage de la violence politique ${ }^{16}$ et de signer le pacte Ajulia Enea qui fait de l'acceptation du jeu démocratique le dénominateur commun du comportement politique. Ce pacte qui dans sa conception visait à désamorcer le "double jeu" d'Herri Batasuna renforce effectivement les tensions qui pèsent sur la coalition. On se trouve en face d'un dilemme "politique idéale/politique réelle", qui explique la complexité des rapports d'Herri Batasuna avec les autres acteurs politiques, mais également avec ETA. La dialectique pratique légale/illégale qui est celle du mouvement basque n'est pas tenable pour Herri Batasuna et il y a à terme un risque d'éclatement par la réintégration d'une partie de la coalition dans le jeu légal, ce qui serait conforme à l'Histoire du mouvement basque scandée par des scissions périodiques.

Les contraintes qui pèsent sur ETA sont égaiement fortes. La seule perspective politique pour l'organisation, puisqu'une victoire militaire reste exclue, est la reprise éventuelle du dialogue d'Alger avec le gouvernement; si le dialogue se renoue, ce sera un événement déterminant car les enjeux politiques sont considérables. Pour l'ETA, il s'agit de forcer le gouvernement à négocier à ses conditions, sous la menace d'attentats, c'est-à-dire de le forcer à reconnaître comme seul véritable partenaire politique au Pays basque : ETA. Avec comme conséquences une décrédibilisation des autres partis politiques basques et un éclatement du pacte Ajulia Enea, autrement dit: tirer un trait sur cinq ans de politique et déstabiliser la scène politique du Pays basque avec en prime une reconnaissance implicite du bien-fondé de la lutte armée. A l'inverse, pour le gouvernement espagnol, les discussions ne doivent être que des conversations techniques gouvernement l'ETA portant sur les conditions d'arrêt de la violence. Ces conversations doivent permettre un retour honorable des etarras dans leur village. La voie est très étroite et les chances de réussite des scénarios de dialogue direct avec le gouvernement espagnol sont réduites, et ce d'autant plus qu'aucun des deux adversaires ne veut courir le risque d'un échec !

Mais il existe un second enjeu capital pour ETA; celui de son enracinement dans la population basque mesuré à travers sa capacité de mobilisation ponctuelle, ou à travers le soutien électoral à Herri Batasuna. Le mythe nationaliste basque cimenté par la violence dont ETA s'institue dépositaire continuera-t-il à fonctionner? Ou la logique du martyre appuyée sur la dénonciation de la répression s'effondrera-t-elle devant les discours plus réalistes du PNV $?^{17}$.

Conjectures

Quelle stratégie va mener ETA ? Deux hypothèses sont envisageables. La première, la plus pessimiste, consiste à penser qu'ETA est restée sur une ligne dure et envisage toujours un scénario militaire selon lequel le gouvernement espagnol cédera à la pression de la violence. Cependant ETA est tout de même consciente que les opérations 
policières de la première partie de l'année, non seulement ont considérablement affaibli son potentiel action - il n'existerait actuellement plus que deux commandos capables d'entreprendre des actions - mais surtout qu'elles ont largement érodé sa crédibilité politique.

La deuxième hypothèse est à l'opposé - c'est la moins coûteuse en terme de vies humaines- elle suppose qu'ETA a pris bonne note de son affaiblissement et que ses dirigeants ont compris qu'ils ne peuvent espérer faire ployer par la menace le gouvernement espagnol. Cette hypothèse part d'un constat : la lutte militaire $a$ échoué car la violence a des effets désastreux sur l'opinion basque : chaque attentat cristallise les positions et bloque un peu plus l'évolution politique. Or, le rapport de force n'est à ce point en faveur de l'État espagnol que parce que celui-ci bénéficie de la légitimité accordée à celui qui est attaqué. Il faut donc modifier la stratégie vers l'indépendance et revenir aux réalités du monde actuel, en passant d'une rhétorique et d'une pratique militaire à une pratique gestionnaire . Si cette analyse ${ }^{18}$ est acceptée par ETA, le problème de l'indépendance basque peut être posé en terme de relais, relais d'ETA par le PNV, seul parti à même de "revisiter" l'indépendance.

C'est dans ce cadre qu'il faut analyser l'échec des deux événements majeurs du printemps et de l'été 92 : les conversations entre le PNV et Herri Batasuna, inaugurées à Bilbao Ie 5juin, dont l'objet étaitjustement d'étudier le moyen de mettre fin à la violence politique au Pays basque, et la proposition de trêve des attentats faite par ETA le 10 juillet.

Les dix rencontres entre le PNV/HB n'ont pas abouti à un accord et sont difficiles à apprécier ; Herri Batasuna, évoluant après la prise de contact sympathique du 10juin, entre la souplesse -séance du 23 juin - et la fermeté - séance du 10 juillet où elle remet en avant l'alternative KAS. Ces variations d'attitudes ${ }^{19}$ peuvent être interprétées comme les signes d'une attitude concertée où les nationalistes soufflent alternativement le chaud et le froid; nous y verrions plutôt les premiers effets de la dislocation d'Herri Batasuna soumise aux contraintes de la dynamique de l'adieu aux $\operatorname{armes}^{20}$.

La proposition de trêve et d'ouverture de négociations faite au gouvernement espagnol le 10juillet par ETA est également délicate à interpréter. Le refus rapide du gouvernement espagnol à cette proposition n'a pas de quoi surprendre puisqu'il ne fait que réaffirmer la position officielle de "non négociation" des autorités, mais il faut rappeler que Ie gouvernement espagnol n'a cessé de garder le contact avec les séparatistes basques et que ce refus officiel ne veut surtout pas dire qu'aucun contact ne soit maintenu avec les séparatistes ${ }^{21}$. La presse espagnole se livre d'ailleurs au petit jeu de savoir qui serait le meilleur négociateur. Le président du gouvernement autonome basque, José Antonio Ardanza, qui est aussi président du Parti national basque, a déclaré quant à lui que cette proposition n'apportait rien de nouveau, alors que le porte-parole du PNV, Joseba Egibar, y voyait un pas positif. Cette apparente opposition peut s'expliquer par une question de personnalité, J. A. Ardanza s'est déjà fait remarquer par des positions très nettes contre ETA, ou plus vraisemblablement elle relève d'une différence de positions institutionnelles: le président du gouvernement autonome ne parle pas de la même manière que le porte-parole d'un parti qui de surcroît est engagé dans une négociation. Mais la position contradictoire du PNV peut aussi s'expliquer par un calcul visant à disloquer Herri Batasuna dont les positions semblent ces derniers mois avec le nouveau climat politique fluctuer entre fermeté et 
conciliation. Alors l'initiative d'ETA correspondrait-elle à une man ?uvre tactique, l'organisation tendant un piège au pouvoir en le mettant en demeure de choisir entre la négociation politique ou la continuation de la violence. En cas de refus de la trêve par le gouvernement, l'organisation lui aurait fait porter la responsabilité politique de la reprise de la violence en l'accusant d'intransigeance, c'est-à-dire en renversant devant la population, selon un mécanisme classique, la causalité. Si le gouvernement avait accepté, l'organisation, en redevenant le seul véritable interlocuteur politique au Pays basque, aurait comblé à peu de frais son déficit de légitimité. Ou, hypothèse inverse, cette initiative est-elle à inscrire dans un véritable changement de stratégie de l'organisation? Il est difficile de trancher, néanmoins, la première hypothèse semble la plus crédible, d'autant que le 18 août, ETA a annoncé dans un communiqué la poursuite de la lutte armée ${ }^{22}$.

Conclusion

Dans la gestion du conflit les lignes s'infléchissent. La police marque des points, et devrait en marquer de plus en plus avec le développement de l'Ertzaintza. Sur le plan politique, le développement du statut d'autonomie et corrélativement la représentativité de plus en plus forte des partis du pacte Ajulia Enea symbolisent la perte d'inf1uence d'ETA comme organisation monopolisant la représentation politique basque. Ne sommes-nous pas à un carrefour pour ETA? Dans une situation de dislocation du projet national devant la montée en puissance de l'autonomie réelle, le recours à la violence aveugle n'est-il pas pour l'organisation - et depuis longtemps - le moyen de voiler un discours en perte de vitesse? A moyen terme, la démarche émotive et chargée de symboles d'ETA est-elle en train de céder le pas devant le réalisme gestionnaire du PNV ? Tel est l'enjeu du débat intrabasque.

\section{NOTES}

1. Couronnée par l'explosion d'une voiture piégée à Madrid le 6 février. Nous remercions l'IHESI et le MRT de nous autoriser à reproduire ce texte tiré d'un rapport de recherche sur "Le terrorisme en Europe à l'horizon 92".

2. Un sénateur de Herri Batasuna, Iñigo, a servi d'intermédiaire entre les industriels et l'ETA pour que les premiers puissent rencontrer le membre d'ETA à qui ils devaient payer l'impôt révolutionnaire. Selon un communiqué du ministère de l'Intérieur basque, Iruin n'aurait pas joué ce rôle par philanthropie, comme le déclare, mais parce qu'il collaborait avec l'ETA. Le réseau a été démantelé par la police autonome basque (Ertzaintza) qui a enregistré clandestinement 200 heures de vidéo démontant le trafic. 3. C'est en 1987 que le parlement basque approuva à l'unanimité le plan de substitution au Pays basque de la police espagnole par Ertzaintza.

4. Déclaration allant dans le même sens Juan-Mariá Atutxa, Ministre de l'intérieur basque, El Pais, 9 février 1992.

5. Voir note 3.

6. Typique de cette attitude, Diaro 16 du 13 février 1992 publie un article à base de révélations policières. Illustré d'une photo, vieille de 12 ans, des membres de la 
direction d'ETA, en cagoule, l'article est agrémenté du commentaire suivant : "2 "encagoulés" que la police dit très proches d'Herri Batsuna ont participé avec "Pakito" (Artapalo) aux dernières réunions de la cúpula d'ETA en France ; La police espagnole suppose qu'ils constituent la "direction interne" d'ETA en Espagne".

7. La presse espagnole annonçait le 13 février 1992 que les ministres de l'Intérieur espagnol et français, José-Luis Corcuera et Philippe Marchand, étaient tombés d'accord d'accord sur le principe d'un accord visant à créer des brigades mixtes en regroupant des policiers français et espagnols et opérant en France. Elle faisait référence à une déclaration sibylline de José-Luis Corcuera lors des funérailles à Murcie de J.L. Faris et à un entretien de Felipe Gonzales au quotidien britannique The Times, que complétaient des "sources autorisées" des deux ministères (El pais du 13, El Sol du 14). La France démentira quinze jours plus tard (Le Monde du 29 février 1992).

8. Urrats Berri - 4 documents de base en basque et en espagnol ont été publiés par Herri Batasuna en novembre 1991 : analyse de la situation politique (41P ;) ; Batasunean gorante : vers un projet commun des abertzales et de la gauche (45 p.); critère d'action politique : la lutte populaire, le combat des idées (65 p.). Le dernier volume porte sur les modifications d'organisation à apporter au mouvement. On peut y ajouter un petit opuscule intitulé : "Politique institutionnelle d'Herri Batasuna" (19 p.) faisant le bilan sur l'activité d'Herri Batasuna en 1991.

9. Le programme d'Herri Batasuna déclare : un peuple, une langue et rappelle qu'il y a deux conditions de base : que le basque soit nécessaire dans tout type de relation sociale, qu'il soit nécessaire sur le territoire basque.

10. Il faudrait de surcroît faire la différence entre l'ensemble KAS (Conseil de coordination patriotique socialiste) composé par ETA, LAB, JARRAI, ASK, et EIZAN et la coalition électorale Herri Batasuna.

11. ETA politico-paramilitaire (ETA-PM).

12. ETA militaire (ETA-M).

13. Plus l'organisation vieillit sans obtenir de succès significatif, plus le profil de guerrier sommaire remplace celui de stratège politico-militaire. On le voit bien dans la succession des $n^{\circ} 1$ de l'ETA. On peut même se demander si à partir d'un certain stade, la violence ne reste pas la seule ressource politique disponible pour se maintenir en place et donner vie à l'organisation.

14. la complexité du traité de Maastricht montre bien comment les problèmes de modification des articulations entre ensembles nationaux sont délicats, car s'y mêlent les questions proprement politiques de souverainté, mais également les intérêts.

15. La position du PNV sur l'indépendance, appelé quelquefois "doctrine Aguire" fut exposée publiquement après la légalisation du parti en 76. Elle insiste sur deux dimensions. D'une part, la fin des Etats-Nations : La Grande-Bretagne, la France, l'Allemagne ne peuvent plus maintenir la plénitude de leur souveraineté", en conséquence de quoi il est logique "que le peuple basque ne tombe pas dans cette structure étatique, dépassé et vieillie". D'autre part, l'égalité entre les peuples européens donc entre la nation basque et les autres nations : "la nation basque pourra à égalité avec les autres nations européennes faire partie de la future Europe si celle-ci est réellement démocratique et respecte la volonté des peuples qui la composent" (citation tirée de El Nacionalismo vasco y la idea de Europa), conférence du 29 mai prononcée par Xavier Arzalloz Antia. Voir aussi l'entretien avec le PNV. 
16. La seule concession sur la lutte armée est la suivante : au sein d'Herri Batasuna cohabitent des personnes ayant une attitude différente sur la lutte armée, y compris celles qui doteraient de l'utilité de son usage dans la conjoncture actuelle.

17. L'enjeu symbolique est clairement ressenti par les acteurs basques, comme l'ont montré à Bilbao les manifestations du 1er et 8 février 1992. Le 1er février, une manifestation organisée par l'organisation "geste pour la paix" (gesto por la paz), avait réuni 25.000 autour d'un "appel à ETA pour qu'il abandonne la lutte armée". Issu d'une coordination créée en 1986, "geste pour la paix" comprend 91 groupes - soit 15000 personnes disséminées dans tout le Pays basque. Chaque fois que la violence politique fait un mort (civil, policier, militaire, etarra), ces groupes se réunissent silencieusement pour protester pendant quinze minutes. Le 8 , une grande manifestation de soutien à ETA organisée par Herri Batasuna lui répondait. A la déclaration du président du gouvernement basque, J.A. Ardanza, recueillie pendant la manifestation du 1er février : " c'est la première fois que cela prend cette envergure, parce que c'est la première fois qu'une manifestation est le produit de la société à travers "geste pour la paix", répond celle de José Idigora, porte-parole de Herri Batasuna disant le 8 février, que le rassemblement du 1er février était un "acte contre ceux qui défendent le droit à l'autodétermination, contre ceux qui jamais n'ont identifié la paix avec la reddition". Dans le discours qui mit fin à la manifestation d'Herri Batasuna, Paxti Zabaleta, membre de la direction d'Herri Batasuna, dénonce les "pactes créés pour couvrir les actions policières, et les associations créés pour attirer les bonnes volontés".

18. Qui est probablement celle qui tiennent les responsables du PNV quand ils discutent avec Herri Batasuna, comme l'indique le communiqué diffusé par le PNV expliquant l'arrêt des conversations avec cette organisation.

19. Auxquelles on pourrait adjoindre l'expulsion du sein d'Herri Batasuna, de Txema Montero, ancien parlementaire européen parce qu'il remettait en question la lutte armée, ou, en sens inverse, les déclarations souples de Patxi Zabaleta, membre de la direction d'Herri Batasuna, sur la possibilité d'une prise de contact entre ETA et le gouvernement.

20. C'est également l'interprétation du PNV.

21. La presse espagnole bruisse de rumeurs sur les contacts qu'aurait eu le gouvernement espagnol avec les séparatistes depuis la fin des négociations d'Alger en avril 1989.

22. Tout récemment, l'attentat du 29 novembre à Madrid relance les spéculations sur le potentiel d'ETA et sur sa stratégie. La baisse de crédibilité ne s'expliquerait pas par un changement de stratégie, mais par les succès policiers. Privée d'une grande partie de son potentiel par l'action policière, ETA n'aurait pas jusqu'à présent réussi à le reconstituer.

\section{RÉSUMÉS}

Depuis la reprise de ses activités, ETA gère son conflit avec Madrid à propos de l'indépendance du Pays basque en mobilisant la population autour de l'exaltation des valeurs nationales et par 
l'usage de la violence. L'auteur oppose à ce modèle militaire, un modèle gestionnaire d'indépendance qui utilise les ressources du statut d'autonomie de la province, et se demande si les événements de 1992 ne vont pas hâter le remplacement du premier modèle par le second.

Since its reactivation, ETA, in its struggle against Madrid for the independence of the Basque country, has mobilised the population by an exaltation of national values and the use of violence. The author opposes this military model to a political one that uses the resources of the provincial statute of autonomy. He envisages that the latter may replace the former as a result of the events of 1992.

INDEX

Index géographique : Espagne, France

Mots-clés : guérillas et organisations clandestines, séparatismes/ autonomismes, violence Thèmes : ETA

\section{AUTEUR}

\section{DANIEL HERMANT}

Professeur agrégé d'histoire, directeur de publication de Cultures \& Conflits. 